\title{
Working memory training using EEG neurofeedback in normal young adults
}

\author{
Shi Xiong ${ }^{\mathrm{a}}$, Chen Cheng ${ }^{\mathrm{a}}$, Xia $\mathrm{Wu}^{\mathrm{a}}$, Xiaojuan Guo ${ }^{\mathrm{a}}$, Li Yao ${ }^{\mathrm{b}}$ and Jiacai Zhang, ${ }^{\mathrm{a},}$ \\ ${ }^{a}$ College of Information Science and Technology, Beijing Normal University, Beijing 100875, China \\ ${ }^{b}$ State Key Laboratory of Cognitive Neuroscience and Learning, Beijing Normal University, Beijing \\ 100875, China
}

\begin{abstract}
Recent studies have shown that working memory (WM) performance can be improved by intensive and adaptive computerized training. Here, we explored the WM training effect using Electroencephalography (EEG) neurofeedback (NF) in normal young adults. In the first study, we identified the EEG features related to WM in normal young adults. The receiver operating characteristic (ROC) curve showed that the power ratio of the theta-to-alpha rhythms in the anterior-parietal region, accurately classified a high percentage of the EEG trials recorded during WM and fixation control (FC) tasks. Based on these results, a second study aimed to assess the training effects of the theta-to-alpha ratio and tested the hypothesis that upregulating the power ratio can improve working memory behavior. Our results demonstrated that these normal young adults succeeded in improving their WM performance with EEG NF, and the pre- and post-test evaluations also indicated that WM performance increase in experimental group was significantly greater than control groups. In summary, our findings provided preliminarily evidence that WM performance can be improved through learned regulation of the EEG power ratio using EEG NF.
\end{abstract}

Keywords: Electroencephalography, neurofeedback, working memory, power spectrum, self-regulation

\section{Introduction}

Working memory (WM) refers to the ability to maintain and manipulate information over short periods of time in the context of concurrent processing or distraction [1,2]. Previous studies have reported that WM capacity is of central importance in a wide range of higher-order cognitive functions, such as reasoning, problem solving, and language comprehension [3-5]. Recently, interest in the enhancement of WM capacity has increased as a result of the success and popularity of WM-training studies [6,7]. WM training was originally used to enhance WM in neuropsychiatric subjects with a WM deficit, such as ADHD [8]. A series of subsequent studies by Jaeggi reported that by performing dual n-back tasks, the effects of WM training also generalized to untrained tasks requiring WM and improved a complex human ability known as fluid intelligence $[9,10]$.

Recently, neurofeedback (NF) has become a popular trend in WM training. Since electroencephalography (EEG) is the most widely used NF signal, NF often refers to EEG biofeedback,

\footnotetext{
${ }^{*}$ Corresponding author: Jiacai Zhang, College of Information Science and Technology, Beijing Normal University, No. 19, Xinjiekouwai St., Haidian District, Beijing 100875, China. Tel.: +86 10 58807856; Fax: +86 10 58800056; E-mail: jiacai.zhang@bnu.edu.cn.
}

0959-2989/14/\$27.50 @ 2014 - IOS Press and the authors. 
in which the brain is taught to modulate excitatory and inhibitory EEG features related to some brain function via self-regulation. Although promising results have been reported for NF training in recent literature reports, there is a lack of a deeper understanding of the effects of NF-based WM training. For example, the EEG feature regulated in NF based WM training has been questioned due to a lack of thorough investigation. In Escolano's study, NF training for WM dealt with enhancement of the upperalpha rhythm in EEG [11]. However, Klimesch has presented evidence that the alpha band is not the sole influence in the EEG oscillations that reflect memory performance, but that the theta oscillations also represent or control WM performance [12]. Furthermore, the efficiency of NF training has seldom been studied. Both computerized behavioral training and NF can improve the capabilities of WM functioning. But the issue of whether NF training efficiently boosts brain functions more than behavioral training through direct neurocognitive modulation of brain regions crucial to WM remains to be investigated.

The aim of the present work was to investigate the application of EEG NF in WM training. First, we compared EEG signals involved in a visual WM task and a fixation control (FC) task and identified the EEG activity, such as the EEG spectrum features that contribute to the discriminate WM tasks from FC tasks on a trial-by-trial basis. Second, we evaluate the NF based WM training gains at the neuroelectric level (the trainability of the WM-specific EEG spectrum features) and at the behavioral level (effect and efficiency on improvement of WM performance).

\section{Materials and methods}

\subsection{Subjects}

17 subjects ( 7 females) participated in the first study to determine the EEG signals that would be used for the NF-based WM training. In the second study, to evaluate the WM training effect and efficiency using EEG NF, we recruited a total of 48 subjects, divided randomly and equally into 4 groups: the NF-training group ( 5 females); the behavioral-training group (6 females); the sham NFtraining group (5 females) and the no-training group ( 7 females).

In both studies, all subjects were healthy, right-handed undergraduate or graduate students from Beijing Normal University, all subjects had normal or corrected-to-normal (with contact lenses) visual acuity and also lacked a history of psychiatric or neurological disease or participated in a similar working memory training experiment.

\subsection{Experimental procedure}

Study I: 17 subjects participated in two types of trials: WM (working memory) and FC (fixation cross) trials. In the WM trials, the subjects performed spatial 2-back tasks (Figure 1). In the 2-back task, the subjects were requested to judge whether the current stimulus was identical to the one displayed two positions back in the sequence. Each WM trial was comprised of $10+2$ stimulus images (4 targets and 6 non-targets). The subjects were required to press specific button (space button on the keyboard) only when the target was visible. During the FC trials, subjects simply remained fixed and pressed a button during each response period. Each stimulus image displayed for 1 second with a 1second inter-stimulus interval (ISI). Each subject performed 12 sessions, including $7 \mathrm{WM}$ sessions and $5 \mathrm{FC}$ sessions in random order. Each WM session contained $6 \mathrm{WM}$ trials with a 7 -second break 
between trials, as did the FC sessions. The response accuracy and time of the button press were recorded in the WM tasks and the feedback to the subject on a trial basis.

Study II: Figure 2 outlines the entire experimental procedure for all 48 subjects in the 4 groups. Subjects in each group began with a pre-test and ended with a post-test. In pre/post-test, all subjects were tested on the 2-back WM tasks as were those in Study I. Each test contained 12 sessions, including $7 \mathrm{WM}$ sessions and $5 \mathrm{FC}$ sessions in random order, identical to Study I. The pre- and posttest lasted for about 30 minutes (excluding breaks). All subjects in three training groups, except those in no-training group, attended one of the training procedures in Figure 2.

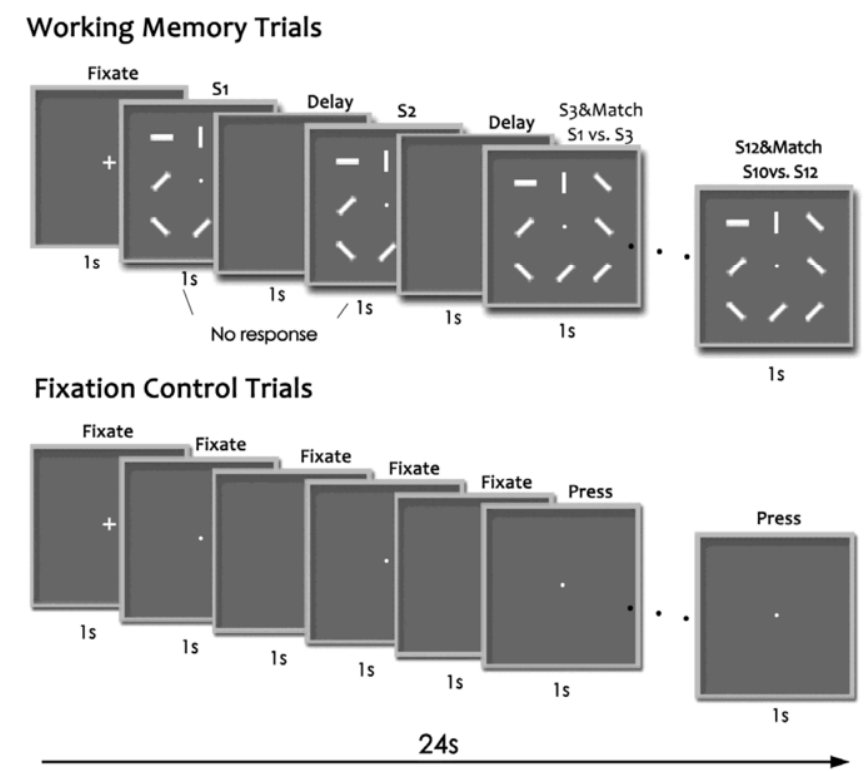

Fig. 1. Experimental Design. Two types of trials were employed: working memory (WM) and fixation control (FC).

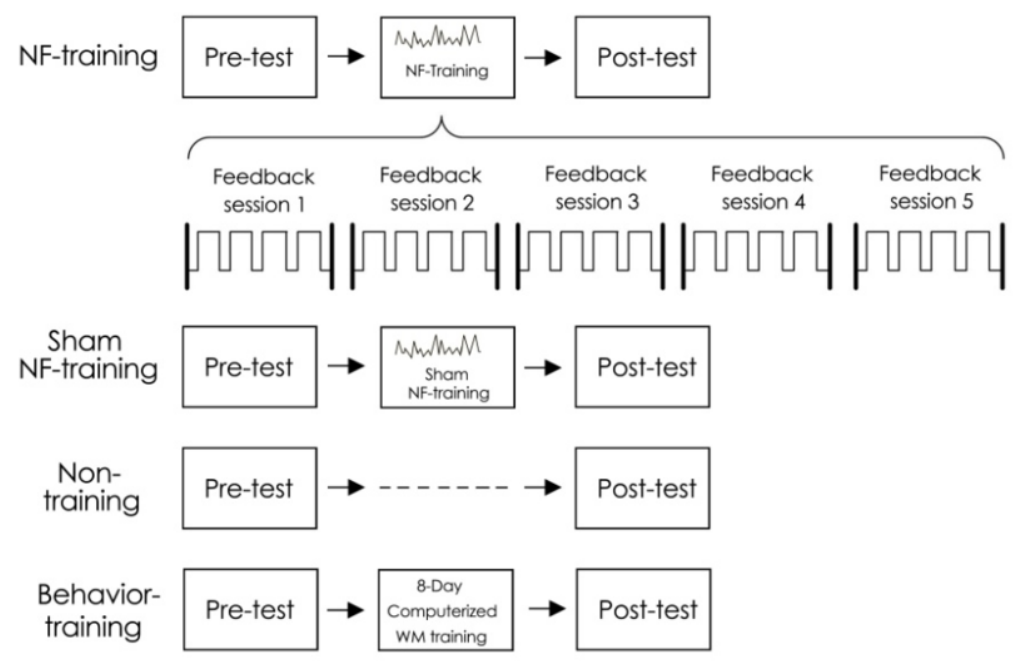

Fig. 2. Experimental Procedure for NF training and control groups in Study II. 

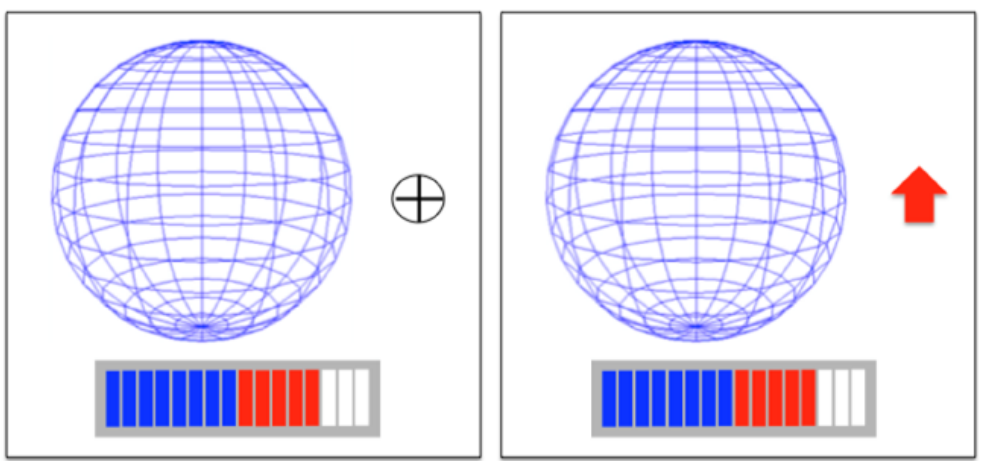

Fig. 3. Video feedback to subjects during NF training. The feedback consisted of a rotating sphere and thermometer bars below it. The rotation speeds of sphere and thermometer level were updated simultaneously during NF training. Bars over the baseline level were colored in red, whereas bars under the baseline level were colored in blue. The baseline level was updated with the average signal of the latest rest block. The regulation and rest blocks were cued with a red arrow (right) or crosshair (left), respectively, next to the globe.

\subsection{Neurofeedback task and control tasks}

NF-training group: the pre-test, NF training and post-test were performed on 3 consecutive days. The NF-training performed $5 \mathrm{NF}$ sessions in one day. Each NF session consisted of 4 regulation blocks of 30 seconds each alternating with 4 rest blocks of 30 seconds each. While in the rest blocks, subjects were instructed to rest and not recall anything about the regulation and try to return their brain activity to the baseline level. The regulation blocks were cued with a red arrow at the thermometer display, whereas during the rest blocks, a crosshair was presented at the same position (Figure 3).

During regulation blocks, subjects were instructed to use a cognitive strategy to persistently increase the level in the thermometer and decrease the rotation speed of the sphere as much as possible. Before formal NF training, the subjects were instructed to use cognitive strategies that would potentially help them to learn to control WM-specific brain activity, such as, lyric rehearsal, the backwards recall of digits or mnemonic associations.

During NF training, continuous EEG was recorded and the EEG feature (theta-to-alpha power ratio) determined in Study I was computed and online feedback was provided to the subjects in the form of a rotating sphere with a delay of approximately $0.5 \mathrm{~s}$, which is the length of the step of the sliding window. The rotation speed of the sphere was constantly updated, i.e., rotation speed increased when the EEG features related to 2-back WM task got smaller and vice versa.

Sham NF training group: subjects completed the same experimental procedure and received the same instructions as the NF training group, but the feedback features were sham NF signals, which were not from their EEG rhythms, but rather from a mixture of noise and EEG signals from other subjects.

Behavioral-training group: subjects attended the computerized WM training (CWMT) training by performing WM tasks (2-back tasks) implemented in a computer program developed for this study. The pre/post-test, as well as the training itself was identical. Moreover, the behavioral training lasted for 8 days without interruption. Training of each day was about 30 min (approximately 5 hours in total).

No-training group: subjects also attended the pre-test and post-test separated by an 8-day interval, and subjects were not trained between the two test sessions. This group served as a control to assess the effects of the repetitive performance of the same WM tasks. 


\subsection{EEG data acquisition and signal processing}

A Biosemi Active Two EEG acquisition system was used to obtain the EEG data. The 64 electrode channels were distributed according to the 10-20 reference placement system. The EEG data were continuously recorded and sampled to $256 \mathrm{~Hz}$. The power of the EEG rhythms, such as theta $(4-7 \mathrm{~Hz})$, alpha $(8-12 \mathrm{~Hz})$, SMR $(12-15 \mathrm{~Hz})$, low beta $(13-20 \mathrm{~Hz})$ and high beta $(20-30 \mathrm{~Hz})$ were computed from the moving averaged EEG power spectra. The EEG data during the pre-test/post-test were analyzed offline using Matlab R2011b, while the EEG data during NF training was analyzed online using Visual $\mathrm{C}++2005$ software was running on a personal computer connected via USB to the Biosemi ActiveTwo.

\section{Results}

\subsection{WM-related, specific EEG rhythms}

The averaged scalp map of theta band between the onsets of the stimuli and responses ( $2 \mathrm{~s})$ across the subjects in the WM and FC condition of Study I was calculated first. We found that the difference of activation between WM and FC tasks is most obvious in the anterior-parietal area. Therefore, in the subsequent analysis, the EEG signals recorded in electrodes $(\mathrm{Fz}, \mathrm{FCz}, \mathrm{Cz}, \mathrm{C} 1$ and $\mathrm{C} 2)$ were selected.

Figure 4 shows that the power of the theta rhythms was larger in the WM trials than in the FC trials, whereas the power of the alpha rhythm was the reverse. These effects on both the theta and the alpha rhythms were confirmed to be significant by statistical testing (pairwise t-test). These findings constitute the most important basis of Study II.

To further examine which rhythm was related to WM function, we exploited alpha rhythm, theta rhythm and the ratio of theta-to-alpha to distinguish WM and FC. As a result, the ratio of theta-toalpha seemed like the best feature for classifying the WM and FC tasks. The optimum threshold was set to the value of 2.20 (marked with a red circle in Figure 5), which classified WM and FC tasks with

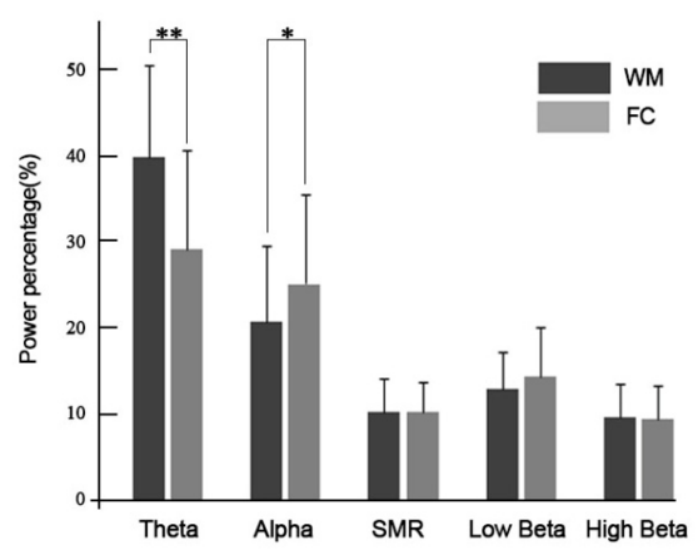

Fig. 4. Power percentage of EEG rhythms from grand average event-related potential (ERP) across 17 subjects and across electrodes $\mathrm{FCz}, \mathrm{Cz}, \mathrm{C} 1, \mathrm{C} 2$ and Fz. Asterisks indicate significant differences between two task conditions. $* * \mathrm{p}<.01, * \mathrm{p}<.05$. 


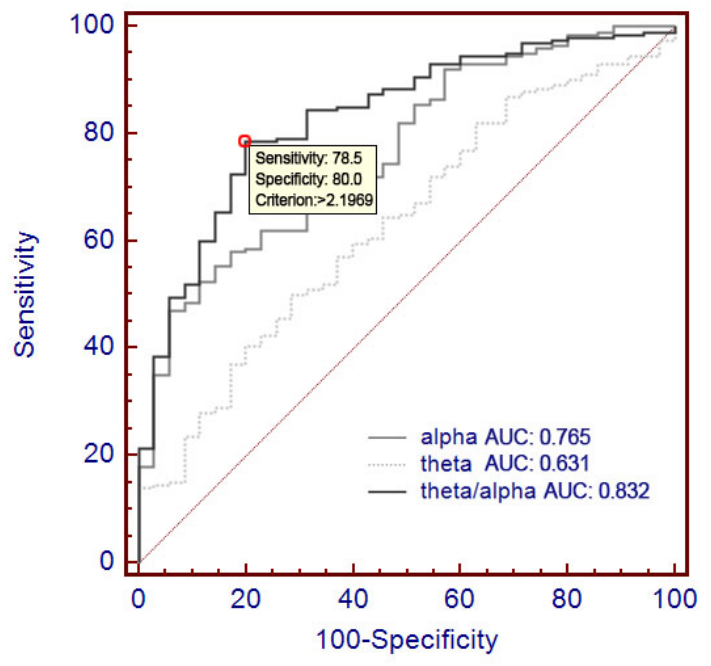

Fig. 5. ROC curve for EEG rhythms and the power ratio of theta-to-alpha rhythm during WM or FC task.

minimum error.

\subsection{Training effects in 2-back task performance}

To assess the change in cognitive performance, measured by the response accuracy and time in 2back tasks, all subjects in Study II were administered a pre-test and post-test. Table 1 shows the changes in the response accuracy and time in the 2-back task in both the pre-test and the post-test.

We first verified the normality of our behavioral data using the Kolmogorov-Smirnov (KS) statistical test. Moreover, our results of one-way ANOVA demonstrate comparable performance of all groups for the WM task at pre-test (accuracy: $F(3,44)<0.184, p>0.906$; response time: $F(3,44)<1.131$, $\mathrm{p}>0.347$ ). Pairwise comparisons between the pre-test and post-test can also be found in Table 1 .

Table 1

Performance of 2-back as function of training effects

\begin{tabular}{|c|c|c|c|c|c|c|c|c|c|c|c|}
\hline & $\begin{array}{l}\text { 2-back } \\
\text { task }\end{array}$ & $\begin{array}{l}\text { Pre-test } \\
\text { Mean }\end{array}$ & SD & Min & Max & $\begin{array}{l}\text { Post-test } \\
\text { Mean }\end{array}$ & SD & Min & Max & $\begin{array}{l}\text { Effect } \\
\text { Size (d) }\end{array}$ & $\begin{array}{l}\text { Pairwise } \\
\text { t-test (p) }\end{array}$ \\
\hline \multirow{2}{*}{ NFT } & Acc $(\%)$ & 74.51 & 15.01 & 44.12 & 91.18 & 90.69 & 4.83 & 82.35 & 97.06 & 1.45 & .006 \\
\hline & $\mathrm{RT}(\mathrm{ms})$ & 3780.2 & 983.4 & 1857.6 & 5361.9 & 2592.6 & 755.6 & 1298.5 & 3560.8 & -1.35 & .001 \\
\hline \multirow{2}{*}{ SNFT } & $\operatorname{Acc}(\%)$ & 76.84 & 16.98 & 50.00 & 94.12 & 81.70 & 12.33 & 58.82 & 94.12 & 0.33 & .325 \\
\hline & $\mathrm{RT}(\mathrm{ms})$ & 3644.5 & 944.1 & 2468.7 & 5137.8 & 3419.9 & 1307.4 & 1711.9 & 5837.0 & -0.20 & .625 \\
\hline \multirow{2}{*}{ BT } & $\operatorname{Acc}(\%)$ & 77.70 & 11.05 & 47.50 & 90.00 & 92.08 & 2.41 & 89.10 & 95.00 & 1.80 & .001 \\
\hline & $\mathrm{RT}(\mathrm{ms})$ & 3856.7 & 1213.1 & 1949.1 & 6017.5 & 1640.3 & 363.5 & 1347.1 & 2179.1 & -2.48 & .785 \\
\hline \multirow{2}{*}{ NT } & Acc $(\%)$ & 77.98 & 7.67 & 61.90 & 88.10 & 82.94 & 7.79 & 73.81 & 95.24 & 0.64 & .041 \\
\hline & RT (ms) & 3218.2 & 1171.3 & 1904.2 & 5349.9 & 2860.3 & 833.2 & 1825.4 & 4853.0 & -0.35 & .001 \\
\hline
\end{tabular}




\section{Discussion}

\subsection{Link between EEG rhythms and working memory}

The results of Study I revealed that the activation regions in WM tasks were mainly located in the frontal and prefrontal areas. These findings support the results of recent studies [13,14], indicating that working memory utilizes the posterior association cortex, which is involved in the storage of sensory information and the prefrontal cortex, which updates the information.

Previous studies have has widely discussed the link between the theta band and WM. Research $[15,16]$ has also illustrated that the EEG patterns associated with the encoding of physics concepts in working memory revealed greater theta activity that was accompanied by the onset of a word stimulus rather than a picture stimulus. According to Norbert, the increase in theta synchronization means enhanced encoding, retrieval, and performance of the central executive and attention control [17]. The present study proved that the improvement of WM was related to the enhancement of theta rhythm and suppression of alpha rhythm. Our results showed that alpha band as well as the theta band was related to WM performance. This was consistent with a previous alpha synchronization study [18].

\subsection{WM improvement and NF-training}

In our study, 2-back tasks were used in the pre-test, post-test and behavioral training. This testing may have added a priming effect to our training effect as the same stimuli lead to task-specific improvement. These increases reflected, at least partly, the priming effect when the post-test task used the same or similar materials as the pre-test task. To clarify the improvement in the primary interest memory process for NF training we compared the gain in accuracy between different groups and found that the improvement of the NF-training group was substantially superior to that of the sham NF- and no-training groups (Table 1).

Another interesting result of the WM training is its transfer effect. Related studies always use a task different from the training task to test the training effects (near-transfer effect) at the same time to avoid the priming effect. For instance, Yvonne Brehmer examined the transfer effect of WM training in sustained attention for healthy older adults [18]. Previous studies conducted by Norbert et al. also proved that WM training could improve intelligence [17]. The hypothesis that by enhancing the thetato-alpha power ratio using NF training improved intelligence was in line with the findings that highly intelligent individuals displayed greater theta synchronization in the early phases of WM task completion [19]. Based on these finding, NF training with theta/alpha enhancement may improve the WM maintenance functions as well as central executive functions and provide a new direction for increasing fluid intelligence.

\section{Conclusion}

This study has described an NF-training regime that employed EEG to improve working memory performance by increasing the theta-to-alpha power ratio in normal young adults. The experimental results from normal young adults showed that five-session NF-training effectively increased the theta/alpha ratio. Furthermore, the enhanced theta/alpha ratio was associated with a significant improvement in behavior performance in WM tasks. In addition, the comparison of training results between NF-training and control groups (sham-NF training, behavioral training and non-training) 
provided promising neurobehavioral evidence that supports the theta/alpha NF-training as a safe, inexpensive, and accessible approach for WM improvement.

\section{Acknowledgement}

This work is supported by 863 Program (2012AA011603) and the Fundamental Research Funds for the Central Universities. This work is also supported by the NSFC Key Program (91320201), Major International (Regional) Joint Research Projects (61210001) and General Program (61375116).

\section{References}

[1] A. Baddeley, Working memory, Science 255 (1992), 556-559.

[2] Y. Brehmer, Working-memory training in younger and older adults: training gains, transfer, and maintenance, Frontiers in Human Neuroscience 6 (2012), 63-69.

[3] T. Nettelbeck, Processing speed, working memory and reasoning ability from childhood to old age, Personality and Indi-vidual Differences 48 (2010), 379-384.

[4] A.R. Conway, Working memory capacity and its relation to general intelligence, Trends in Cognitive Sciences 7 (2003), $547-552$.

[5] V. Camos, Low working memory capacity impedes both efficiency and learning of number transcoding in children, Journal of Experimental Child Psychology 99 (2008), 37-57.

[6] H. Takeuchi, Training of working memory impacts structural connectivity, The Journal of neuroscience 30 (2010), $3297-3303$

[7] A. Baddeley, Working memory: Looking back and looking forward, Nature Reviews Neuroscience 4 (2003), 829-839.

[8] T. Klingberg, Training of working memory in children with ADHD, Journal of Clinical and Experimental Neuropsychology 24 (2002), 781-791.

[9] S.M. Jaeggi, The relationship between n-backperformance and matrixreasoning implications for training and transfer, Intelligence 38 (2010), 625-635.

[10] S.M. Jaeggi, Improving fluid intelligence with training on working memory, Proc. Natl. Acad. Sci. 105 (2008), 68296834.

[11] Escolano, EEG-based upper alpha neurofeedback training improves working memory performance, 33rd Annual International Conference of the IEEE Eng. Med. Biol. Soc. 33 (2011), 2327-2730.

[12] W. Klimesc, EEG alpha and theta oscillations reflect cognitive and memory performance: A review and analysis, Brain Research Reviews 29 (1999), 169-195.

[13] V. Stein, Different frequencies for different scales of cortical integration from local gamma to long range alpha theta synchronisation, Int. J. Psychophysiol. 38 (2000), 301-313.

[14] D. Vernon, The effect of training distinct neurofeedback protocols on aspects of cognitive performance, International Journal of Psychophysiology 47 (2003), 75-85.

[15] K. Lai, Encoding of Physics Concepts: Concreteness and presentation modality reflected by human brain dynamics, PloS One 7 (2012), e41784.

[16] R.J. Addante, Prestimulus theta activity predicts correct source memory retrieval, Proc. Natl. Acad. Sci. 108 (2011), 10702-10707.

[17] N. Jaušovec and K. Jaušovec, Working memory training: Improving intelligence-changing brain activity, Brain and Cognition 79 (2012), 96-106.

[18] Y. Brehmer, Neural correlates of training-related working-memory gains in old age, NeuroImage 58 (2011), 1110-1120.

[19] P. Sauseng, EEG alpha synchronization and functional coupling during top-down processing in a working memory task, Hum. Brain. Mapp. 26 (2005), 148-202. 